\title{
Limit equilibrium payoffs in Stochastic Games
}

\author{
Jérôme Renault, Bruno Ziliotto ${ }^{\dagger}$
}

\begin{abstract}
We study the limit of equilibrium payoffs, as the discount factor goes to one, in nonzero-sum stochastic games. We first show that the set of stationary equilibrium payoffs always converges. We then provide 2-player examples in which the whole set of equilibrium payoffs diverges. The construction is robust to perturbations of the payoffs, and to the introduction of normal-form correlation.
\end{abstract}

Keywords: Stochastic games, Limit equilibrium payoffs.

\section{Introduction}

Stochastic games were introduced by Shapley [21] and generalize repeated games: the payoff functions of the players evolve from stage to stage, and depend on a state variable observed by the players, whose evolution is affected by the players' actions. Actions are revealed at the end of each stage. We denote by $E_{\delta}$, resp. $E_{\delta}^{\prime}$, the set of Nash equilibrium payoffs, resp. sequential equilibrium payoffs, of the $\delta$-discounted game, and we write $E_{\infty}$ for the set of uniform equilibrium payoffs of the dynamic game. We focus on studying the limit, for the Hausdorff distance, of $E_{\delta}$ and $E_{\delta}^{\prime}$ as players become extremely patient, i.e. as the discount factor goes to one.

In the particular case of a standard repeated game, the dynamic interaction simply consists of the repetition of a given one-shot game. The standard Folk theorems hold, with pioneering work from the seventies by Aumann and Shapley [2], and Rubinstein [20]. Regarding sequential equilibrium payoffs, the Folk theorem of Fudenberg and Maskin [8] implies that for generic payoff functions, $E_{\delta}^{\prime}$ converges to the set of feasible and individually rational payoffs of the oneshot game, and Wen [28] showed how to adapt the notion of individually rational payoffs to obtain a Folk theorem without genericity assumption. Without assumptions on the payoffs, $E_{\infty}$ coincides with the set of feasible and individually

*TSE (Université Toulouse 1 Capitole), 21 allée de Brienne, 31000 Toulouse, France.

${ }^{\dagger}$ CNRS, CEREMADE, Université Paris Dauphine, PSL Research Institute, Place du Maréchal de Lattre de Tassigny, 75016 Paris. Both authors gratefully acknowledge the support of the Agence Nationale de la Recherche, under grant ANR JEUDY, ANR-10-BLAN 0112. This work was also supported by ANR-3IA Artificial and Natural Intelligence Toulouse Institute (first author) and by the PEPS "Jeunes Chercheurs" (second author). 
rational payoffs of the one-shot game, and the set of Nash equilibrium payoffs $E_{\delta}$ also converges to this set (see Sorin [24]). These results have been generalized in many ways to games with imperfectly observed actions (see e.g. Abreu et al. [1], Fudenberg and Levine [5], Fudenberg, Levine and Maskin [6], Fudenberg et al. [7], Lehrer [12, 13, 14], or Renault and Tomala [18, 19]), but a full overview of that literature is beyond the scope of the present paper.

In zero-sum stochastic games, Bewley and Kohlberg [3] proved the existence of the limit of the discounted value (hence of $E_{\delta}$ and $E_{\delta}^{\prime}$ ) when $\delta$ goes to one. An example from Sorin [23] shows that in the general-sum case, $\lim _{\delta \rightarrow 1} E_{\delta}$ and $E_{\infty}$ may be nonempty and disjoint. Vieille $[25,26]$ proved that for 2-player games $E_{\infty}$ is always nonempty, that is, there exists a uniform equilibrium payoff ${ }^{1}$.

Regarding discounted equilibrium payoffs in stochastic games, several Folk theorems have been proved under various assumptions. Dutta [4] assumes that the set of long-run feasible payoffs is independent of the initial state, has full dimension, and that minmax long-run payoffs do not depend on the initial state either. Fudenberg and Yamamoto ${ }^{2}$ [9] assume that the stochastic game is irreducible (all players but one can always drive the current state where they want, possibly in many stages, with positive probability). Hörner et al. [10] generalize the recursive methods of [6] to compute a limit equilibrium set in stochastic games with imperfect public monitoring, when this limit set does not depend on the initial state (this is the case when the Markov chain induced by any Markov strategy profile is irreducible).

All the above assumptions require that the stochastic game does not depend too much on the initial state, and in particular rule out the existence of multiple absorbing states ${ }^{3}$ with different equilibrium payoffs. We believe that it is also important to study stochastic games in which the actions taken can have irreversible effects on future plays. This is the case in many situations, for example in stopping games, in which players only act once and have to decide when to do so, or when the actions represent investment decisions, or extractions of exhaustible resources.

Regarding stochastic games with finitely many states and actions, the contribution of our paper is twofold. First, we prove that the set of stationary ${ }^{4}$ equilibrium payoffs of the discounted game always converges to a nonempty set. As a consequence, there always exists a selection of $E_{\delta}^{\prime}$ which converges. Secondly, we show that the convergence property cannot be extended to the Nash or sequential equilibrium payoffs sets, by providing the first examples of 2-player stochastic games in which neither $E_{\delta}$ nor $E_{\delta}^{\prime}$ converges: the limit of the equilib-

\footnotetext{
${ }^{1}$ The generalization of this result to games involving more than two players is a well-known open question in dynamic games.

${ }^{2}$ Fudenberg and Yamamoto [9], as well as Hörner et al. [10], address the more general case of imperfect public monitoring.

${ }^{3}$ When an absorbing state is reached, the play will remain forever in that state, no matter the actions played.

${ }^{4}$ A stationary strategy of a player selects after every history a mixed action which only depends on the current state.
} 
rium set may simply not exist in a stochastic game. Thus, it is out of reach to prove a general Folk theorem in stochastic games, in the sense of characterizations of $\lim _{\delta \rightarrow 1} E_{\delta}$ or $\lim _{\delta \rightarrow 1} E_{\delta}^{\prime}$ without further assumptions, such as irreducibility. Our first example is robust to the introduction of normal-form correlation ${ }^{5}$, and our second example is robust to small perturbations of the payoffs.

Section 1 presents the model of stochastic games and our results. Section 2 is dedicated to the proof of the main result, and Section 3 states two open questions.

Notations : $\mathbb{N}, \mathbb{N}^{*}$ and $\mathbb{R}$ respectively denote the sets of nonnegative integers, positive integers and real numbers. All limits of sets in the paper are taken with respect to the Hausdorff distance between non-empty compact subsets of an Euclidean space : $d(A, B)=\max \left\{\max _{a \in A} d(a, B), \max _{b \in B} d(b, A)\right\}$. The notation $d(A, B) \leq \varepsilon$ means that: every point in $A$ is at most distant of $\varepsilon$ from a point in $B$, and conversely.

\section{$1 \quad$ Model and results}

We consider a 2-player stochastic game. Let $K, I$ and $J$ respectively be the finite sets of states, actions for player 1 and actions for player 2 . Let $k_{1}$ in $K$ be the initial state, $u_{1}$ and $u_{2}$ be the state dependent utility functions from $K \times I \times J$ to $\mathbb{R}$, and finally $q$ be the transition function from $K \times I \times J$ to $\Delta(K)$, which is the set of probabilities over $K$. At every period $t \geq 1$ players first learn the current state $k_{t} \in K$ and simultaneously select actions $i_{t} \in I$ and $j_{t} \in J$. These actions are then publicly observed, the stage payoffs are $u_{1}\left(k_{t}, i_{t}, j_{t}\right)$ for player 1 and $u_{2}\left(k_{t}, i_{t}, j_{t}\right)$ for player 2 , a new state $k_{t+1}$ is selected according to the distribution $q\left(k_{t}, i_{t}, j_{t}\right)$, and the play goes to the next period. Given a discount factor $\delta$ in $[0,1)$, the $\delta$-discounted stochastic game is the infinite horizon game where player 1 and player 2's payoffs are respectively $(1-\delta) \sum_{t=1}^{\infty} \delta^{t-1} u_{1}\left(k_{t}, i_{t}, j_{t}\right)$ and $(1-\delta) \sum_{t=1}^{\infty} \delta^{t-1} u_{2}\left(k_{t}, i_{t}, j_{t}\right)$.

We respectively denote by $E_{\delta}, E_{\delta}^{\prime}$ and $E_{\delta}^{\prime \prime}$ the sets of Nash equilibrium payoffs, subgame-perfect ${ }^{6}$ equilibrium payoffs and stationary equilibrium payoffs of the $\delta$ discounted stochastic game. Standard fixed-point arguments show the existence of a stationary equilibrium in this game, and we have $\emptyset \neq E_{\delta}^{\prime \prime} \subset E_{\delta}^{\prime} \subset E_{\delta}$. We are mainly interested in the asymptotic behavior of these sets when players become more and more patient, i.e. we focus on the existence of their limit when $\delta$ goes to 1 .

We will also briefly consider the set $E_{\infty}$ of uniform equilibrium ${ }^{7}$ payoffs of the

\footnotetext{
${ }^{5} \mathrm{~A}$ normal-form correlation device can send one message to each player before the game starts.

${ }^{6}$ or equivalently here, sequential equilibrium, or equivalently perfect public equilibrium, as defined in [6] for repeated games with perfect public monitoring and extended to stochastic games in $[9]$.

${ }^{7}$ Throughout the paper, we say that a vector $u$ in $\mathbb{R}^{2}$ is a uniform equilibrium payoff if for all $\varepsilon>0$, there exists a strategy profile such that for all large enough discount factors, the profile
} 
stochastic game.

In the zero-sum case, Shapley [21] proved that the value $v_{\delta}$ exists and players have stationary optimal strategies, hence $E_{\delta}=E_{\delta}^{\prime}=E_{\delta}^{\prime \prime}$ is a singleton. Bewley and Kohlberg [3] proved the convergence of $v_{\delta}$ (hence, of $E_{\delta}$ and $E_{\delta}^{\prime}$ ) using algebraic arguments. Our first result shows how the Bewley Kohlberg result extends to general-sum games. Though it stems directly from previous results of the literature ([17] and [11]), it did not appear explicitly before.

Proposition 1.1. There exists a non-empty compact set $E$ such that $E_{\delta}^{\prime \prime} \underset{\delta \rightarrow 1}{\longrightarrow} E$.

Notice that in the case of repeated games (a single state), $E$ reduces to the set of mixed Nash equilibrium payoffs of the one-shot game, hence it may not be convex. The proof largely relies on the semi-algebraicity of the set of discount factors and associated stationary equilibria and payoffs. As stated here, it holds for any 2-player stochastic game with finitely many states and actions, but the proof easily extends to the $n$-player case.

Proof of Proposition 1.1: Recall that a subset $A$ of the Euclidean space $\mathbb{R}^{N}$ is said to be semi-algebraic if it is defined by a finite number of polynomial inequalities, i.e. if $A$ is a finite union of sets, each of these sets being defined as the conjunction of finitely many weak or strict polynomial inequalities. The following proposition is a direct consequence of the main theorem in [11] :

Proposition 1.2. Let $\left(W_{\delta}\right)_{\delta \in[0,1)}$ be a family of non-empty compact subsets of the Euclidean space $\mathbb{R}^{N}$. Assume that $\left\{(\delta, x) \mid \delta \in[0,1), x \in W_{\delta}\right\}$ is a bounded semi-algebraic subset of $\mathbb{R}^{N+1}$. Then when $\delta$ goes to one, $W_{\delta}$ converges for the Hausdorff distance to a non-empty compact subset $W$ of $\mathbb{R}^{N}$.

Let now $W$ be the set of $(\delta, x, y, r) \in[0,1) \times\left(\mathbb{R}^{I} \times \mathbb{R}^{J} \times \mathbb{R}^{2}\right)^{K}$ such that $(x, y)$ is a stationary equilibrium in $\Gamma_{\delta}$, and $r$ is the associated equilibrium payoff. It is well known (see e.g. [17, Example E.7 p.63]) that $W$ is semi-algebraic. For $\delta \in[0,1)$, let $W_{\delta}:=\left\{(x, y, r) \in\left(\mathbb{R}^{I} \times \mathbb{R}^{J} \times \mathbb{R}^{2}\right)^{K} \mid(\delta, x, y, r) \in W\right\}$. The set $W_{\delta}$ is non-empty and compact. Applying the proposition, we deduce that $\left(W_{\delta}\right)$ converges when $\delta$ goes to 1 . In particular, $\left(E_{\delta}^{\prime \prime}\right)$ converges.

Remark 1.3. As a consequence, one can construct a selection of $\left(E_{\delta}^{\prime}\right)_{\delta}$ which converges, i.e. it is possible to select, for each discount $\delta$, a perfect equilibrium payoff $x_{\delta}$ of the corresponding game in a way such that $x_{\delta}$ has a limit when $\delta$ goes to one.

This fact can also be easily deduced from [15, Chapter 7, Lemma 2.2] and [16, Theorem 5], who proved the existence of a semi-algebraic selection of $E_{\delta}^{\prime \prime}$. Because

is a $\varepsilon$-Nash equilibrium of the discounted game with payoff $\varepsilon$-close to $u$ (see [24], [15] or [26] for related definitions). 
payoffs are bounded, this selection converges. A similar result has been used in [22, p.685] and [25, p.74]. It can not be extended to stochastic games with compact action spaces, as shown by a zero-sum example by Vigeral [27] where the value $v_{\delta}$ does not converge.

It is natural to ask if the convergence property of Theorem 1.1 also holds for $E_{\delta}$ and $E_{\delta}^{\prime}$. We conclude this section by providing the first examples of stochastic games where these sets of equilibrium payoffs diverge.

Theorem 1.4. The two following assertions hold:

1. There exists a 2-player stochastic game where neither $E_{\delta}$ nor $E_{\delta}^{\prime}$ converges, and which is robust to normal-form correlation.

2. There exists a 2-player stochastic game where neither $E_{\delta}$ nor $E_{\delta}^{\prime}$ converges, and which is robust to perturbations of the payoffs.

\section{Proof of Theorem 1.4}

To prove this theorem, we first present a simple example (Example 2.1 below) where $E_{\delta}$ and $E_{\delta}^{\prime}$ diverge, and which is robust to the introduction of normal-form correlation. We then provide a more elaborate example (Example 2.5) which is robust to perturbations of the payoffs.

Example 2.1. Consider the following stochastic game.

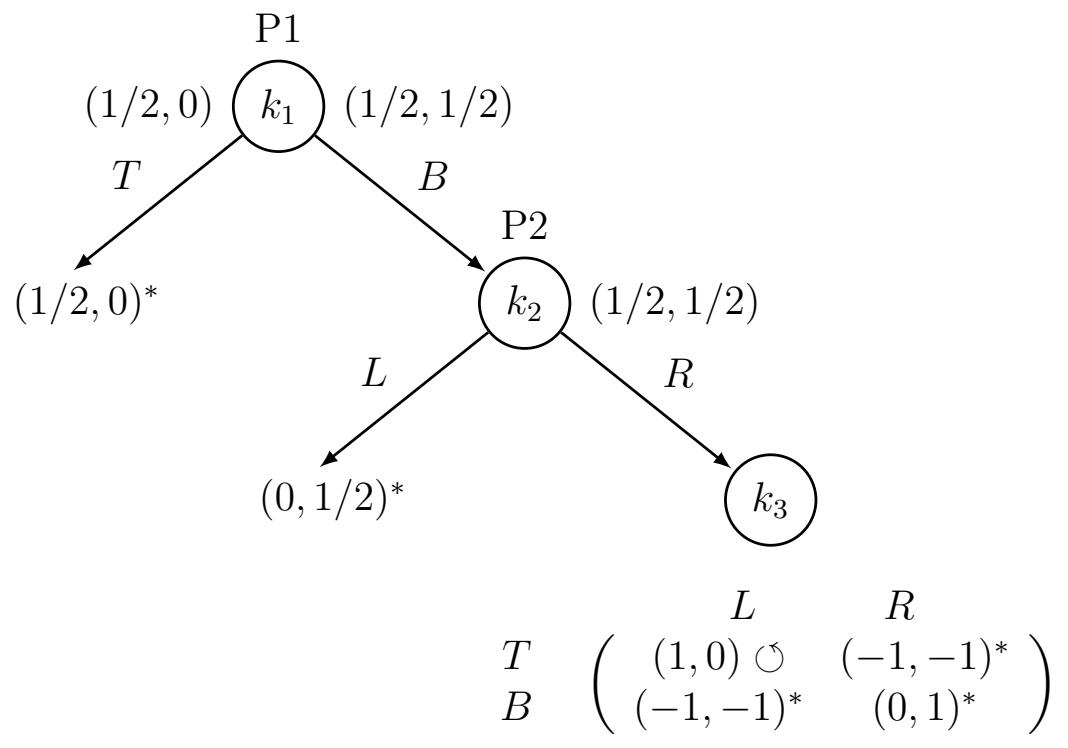

There are 7 states: $k_{1}$ (the initial state), $k_{2}, k_{3}$ and 4 absorbing states: $(1 / 2,0)^{*}$, $(0,1 / 2)^{*},(-1,-1)^{*}$ and $(0,1)^{*}$. When an absorbing state $(a, b)^{*}$ is reached, the 
game stays there forever and at each stage the payoffs to player 1 and player 2 are respectively $a$ and $b$. The sets of actions are $I=\{T, B\}$ for player 1 and $J=\{L, R\}$ for player 2 . The transition from state $k_{1}$ only depends on player 1 's action, as specified in the above figure. Similarly, the transition from state $k_{2}$ only depends on player 2 's action. If in state $k_{3}$ the action profile $(T, L)$ is played, the vector payoff is $(1,0)$ and the play remains in $k_{3}$. To conclude the description, we have to specify the payoffs in states $k_{1}, k_{2}$, and $k_{3}$. The payoff in $k_{1}$ is $(1 / 2,0)$ if $T$ is played and $(1 / 2,1 / 2)$ if $B$ is played. The payoff in state $k_{2}$ does not depend on the actions played and is $(1 / 2,1 / 2)$, and the payoffs in state $k_{3}$ are simply given by the bimatrix $\left(\begin{array}{cc}(1,0) & (-1,-1) \\ (-1,-1) & (0,1)\end{array}\right)$.

For each discount factor, it is clear that $(1 / 2,0)$ is in $E_{\delta}$, and the question is whether there are other equilibrium payoffs, for instance $(1 / 2,1 / 2)$.

Let's first consider any $\delta$ in $[0,1)$, and a Nash equilibrium $(\sigma, \tau)$ of the $\delta$ discounted stochastic game with equilibrium payoff $(x, y)$. Because Player 1 can play $T$ in the initial state, we have $x \geq 1 / 2$. Because the sum of payoffs never exceeds 1 , we have $x+y \leq 1$. Assume now that under $(\sigma, \tau)$, the state $k_{3}$ has positive probability to be reached, and denote by $\left(x_{3}, y_{3}\right)$ the discounted payoffs induced by $(\sigma, \tau)$ given that $k_{3}$ is reached. We have $x_{3} \geq 1 / 2$, because player 1 will not accept to play $B$ at $k_{1}$ if he obtains a payoff lower than $1 / 2$ afterwards. Similarly, $y_{3} \geq 1 / 2$. Since $x_{3}+y_{3} \leq 1$, we get $x_{3}=y_{3}=1 / 2$, so $(1 / 2,1 / 2)$ is an equilibrium payoff of the reduced stochastic game :

$$
\begin{aligned}
& T \\
& B
\end{aligned} \quad\left(\begin{array}{cc}
L & R \\
(1,0) \circlearrowleft & (-1,-1)^{*} \\
(-1,-1)^{*} & (0,1)^{*}
\end{array}\right)
$$

The unique way to obtain $(1 / 2,1 / 2)$ as a feasible payoff in the reduced game is to play first $(T, L)$ for a certain number of periods $N$, then $(B, R)$ at period $N+1$. Indeed, the sum of players' stage payoffs is always not greater than 1 . Thus, if a strategy profile is such that $(T, L)$ or $(B, R)$ is played at some stage of the game, then it can not achieve $(1 / 2,1 / 2)$. Given $\delta$, the integer $N$ has to satisfy $(1-\delta) \sum_{t=1}^{N} \delta^{t-1}=1 / 2$, that is $\delta^{N}=\frac{1}{2}$. If no such integer $N$ exists, we obtain that $(1 / 2,1 / 2)$ is not an equilibrium payoff of the reduced game. Therefore, under $(\sigma, \tau)$, state $k_{3}$ has zero probability to be reached, which implies that $x=1 / 2$ and $y=0$.

Define $\Delta_{1}$ as the set of discount factors of the form $\delta=\left(\frac{1}{2}\right)^{1 / N}$, where $N$ is a positive integer, and $\Delta_{2}=[0,1) \backslash \Delta_{1}$. We have obtained:

Lemma 2.2. For all $\delta$ in $\Delta_{2}, E_{\delta}=E_{\delta}^{\prime}=\{(1 / 2,0)\}$.

Consider now $\delta$ in $\Delta_{1}$, and $N$ such that $\delta^{N}=\frac{1}{2}$. The pure strategy profiles where : $T$ is played at stage $1, R$ is played at stage $2,(T, L)$ is played from stage 3 to stage $N+2$, and $(B, R)$ is played at stage $N+3$, form a subgameperfect Nash equilibrium of the $\delta$-discounted game with payoff $(1 / 2,1 / 2)$. By mixing between $T$ and $B$ in $k_{1}$, it is then possible to obtain any point $(1 / 2, y)$, 
with $0 \leq y \leq 1 / 2$, as an equilibrium payoff. No other point can be obtained, because in every equilibrium, the vector payoff conditional on $k_{3}$ being reached, is $(1 / 2,1 / 2)$. We have obtained :

Lemma 2.3. For all $\delta$ in $\Delta_{1}, E_{\delta}=E_{\delta}^{\prime}=\{1 / 2\} \times[0,1 / 2]$.

Because both $\Delta_{1}$ and $\Delta_{2}$ contain discount factors arbitrarily close to 1 , lemmas 2.2 and 2.3 establish that neither $E_{\delta}$ nor $E_{\delta}^{\prime}$ converge.

Let us consider now normal-form correlated equilibrium payoffs, i.e. Nash equilibrium payoffs of games where the players may initially receive private signals independent of the payoffs. For $\delta$ in $\Delta_{2}$, the proof of Lemma 2.2 applies and the set of normal-form correlated equilibrium payoffs is again the singleton $\{(1 / 2,0)\}$. Consequently, the set of normal-form correlated equilibrium payoffs can not converge when the discount factor goes to one.

Remark 2.4. For any positive integer n, one can also consider the set of Nash equilibrium payoffs $E_{n}$ and subgame-perfect equilibrium payoffs $E_{n}^{\prime}$ of the n-period stochastic game, where the overall payoff is defined as the arithmetic average of the stage payoffs. When $n$ is even, the unique way to obtain $(1 / 2,1 / 2)$ as a feasible payoff in the reduced game is to play first $(T, L)$ for $n / 2$ stages, then $(B, R)$ at period $n+1$. When $n$ is odd, $(1 / 2,1 / 2)$ is not a feasible payoff of the reduced game. Thus, similar arguments show that in Example 2.1, we have $E_{n}=E_{n}^{\prime}=\{1 / 2\} \times[0,1 / 2]$ for $n$ even, and $E_{n}=E_{n}^{\prime}=\{(1 / 2,0)\}$ for $n$ odd. Therefore, $E_{n}$ and $E_{n}^{\prime}$ also do not converge when $n$ goes to infinity. As for the set of uniform equilibrium payoffs, one can show that $E_{\infty}=\{(1 / 2,0)\}$.

Notice that an important feature of the reduced game at state $k_{3}$ is that there is (at most) a unique way to obtain the payoff $(1 / 2,1 / 2)$. As soon as one perturbs the payoffs, this property disappears, and Example 2.1 is not robust to perturbations of the payoffs of the stochastic game.

Example 2.5. Let $\left(a_{1}, a_{2}\right) \in[0,1 / 2]^{2},\left(b_{1}, b_{2}, d_{1}, d_{2}\right) \in[-5,-10]^{4},\left(c_{1}, c_{2}\right) \in$ $[0,1)^{2}$. The stochastic game is represented by the following matrix:

$$
\left.\begin{array}{ccc} 
& L & R \\
M & \left(a_{1}, a_{2}\right) & \left(b_{1}, b_{2}\right)^{*} \\
B & (1,1)^{*} & \left(c_{1}, c_{2}\right)^{*} \\
\left(d_{1}, d_{2}\right)^{*} & (3,0)^{*}
\end{array}\right)
$$

This is an absorbing game, in which each player has only one action which does not lead to an absorbing state. Thus, for all $\delta \in[0,1), E_{\delta}=E_{\delta}^{\prime}$.

Let $\Delta_{1}:=\left\{\delta \in[0,1) \mid \exists N \in \mathbb{N}^{*},\left(1-\delta^{N}\right) a_{1}+3 \delta^{N}=1\right\}=\left\{\left(\frac{1-a_{1}}{3-a_{1}}\right)^{1 / N}, N \in \mathbb{N}^{*}\right\}$, and $\Delta_{2}:=[0,1) \backslash \Delta_{1}$.

Lemma 2.6. For all $\delta \in \Delta_{1},\{1\} \times[1 / 2,1] \subset E_{\delta}$. For all $\delta \in \Delta_{2}$, $E_{\delta} \cap\{\mathbb{R} \times[1 / 2,3 / 4]\}$ is at most a singleton. 
Consider $\delta \in \Delta_{1}$, and let us prove that $\{1\} \times[1 / 2,1] \subset E_{\delta}$. Let $N \in \mathbb{N}^{*}$ such that $\left(1-\delta^{N}\right) a_{1}+3 \delta^{N}=1$. Given some $\alpha \in[0,1]$, consider the following strategy profile: at stage 1 , Player 1 plays $T$ with probability $\alpha$, and $M$ with probability $1-\alpha$, while Player 2 plays $L$. Then, between stage 2 and $N$, players play $(T, L)$, and at stage $N+1$, players play $(B, R)$. This is a Nash equilibrium. Indeed, any deviation of a player at stage 2 leads to an absorbing state with worse payoff. If at stage 1 , Player 1 deviates and plays $B$, he gets worse than 1 . If he plays $T$ or $M$, he gets payoff 1 . And for Player 2 , playing $R$ at stage 1 is not profitable. Under this Nash equilibrium, Player 1 gets payoff 1 , and Player 2 gets payoff $\alpha\left(1-\delta^{N}\right) a_{2}+(1-\alpha)=2 \alpha a_{2}\left(3-a_{1}\right)^{-1}+1-\alpha$. Because $2 a_{2}\left(3-a_{1}\right)^{-1} \in[0,1 / 2]$, we have $\{1\} \times[1 / 2,1] \subset E_{\delta}$.

Consider now $\delta \in \Delta_{2}$, and let us prove that there is at most one Nash equilibrium under which Player 2's total discounted payoff lies in $[1 / 2,3 / 4]$. Let $(\sigma, \tau)$ be a Nash equilibrium. If Player 2 always play $L$, then Player 1 plays $M$ at stage 1 , and Player 2's total discounted payoff is $0 \notin[1 / 2,3 / 4]$. Assume now that there exists some stage such that Player 2 plays $R$ with positive probability, and that at stage 1 , Player 1 plays $T$ with positive probability.

Denote by $m_{0}$ the first stage where Player 2 plays $R$ with positive probability. We claim that for all $m \in\left\{2, \ldots, m_{0}-1\right\}$, at stage $m$, Player 1 plays $T$ with probability 1. Indeed, otherwise consider the first stage $m \in\left\{2, \ldots, m_{0}-1\right\}$ where Player 1 plays either $M$ or $B$ with positive probability. At stage $m$, Player 2 plays $L$, thus Player 1 plays $B$ with probability 0 and $M$ with positive probability. This implies that the normalized continuation payoff of Player 1 from stage $m$ is at most 1 . Because Player 1 gets a payoff strictly smaller than 1 before stage $m$, his total payoff is strictly smaller than 1 . Thus, playing $M$ at stage 1 is a profitable deviation.

Denote respectively $x_{1}, x_{2}$ and $x_{3}$ the probability that at stage $m_{0}$, Player 1 plays $T, M$ and $B$, and denote respectively $y_{1}$ and $y_{2}$ the probability that at stage $m_{0}$, Player 2 plays $L$ and $R$.

Case 1. $y_{2}=1$

At stage $m_{0}$, the game is not finished, thus $x_{3}=1$. Now we discriminate between the two following subcases:

Subcase 1. At stage 1, Player 1 plays $M$ with probability 0 . In this subcase, the total payoff for Player 2 is smaller than $a_{2} \leq 1 / 2$. Consequently, the associated Nash equilibrium payoff does not lie in $\mathbb{R} \times[1 / 2,3 / 4]$.

Subcase 2. At stage 1, Player 1 plays $M$ with some positive probability $\alpha \in$ $(0,1]$. In this subcase, at stage 1 , Player 1 is indifferent between playing $T$ and $M$. Because action $M$ gives a total payoff equal to 1 , we have $\left(1-\delta^{m_{0}-1}\right) a_{1}+3 \delta^{m_{0}-1}=$ 1 , which is a contradiction since $\delta$ lies in $\Delta_{2}$.

Case 2. $y_{2}<1$ 
First, note that Player 1's total discounted payoff lies in $\left[c_{1}, 3\right]$. We claim that $x_{3}>0$. Indeed, otherwise playing $L$ at stage $m_{0}$ would be a profitable deviation for Player 2. Consequently, we have $d_{1} y_{1}+3\left(1-y_{1}\right) \geq c_{1}$. Let us prove that $x_{1}=0$. Indeed, if this was not the case, we would have $3 y_{1}+b_{1}\left(1-y_{1}\right) \geq c_{1}$. Together with the previous inequality, this implies $y_{1}\left(-9 / b_{1}+d_{1}\right) \geq\left(-3 / b_{1}+1\right) c_{1}$. The left-hand side is strictly negative, while the right-hand side is non negative : this is a contradiction, and consequently $x_{1}=0$. Thus, at stage $m_{0},(\sigma, \tau)$ induces a Nash equilibrium in the following one-shot game:

$$
\begin{array}{ccc} 
& L & R \\
B & \left(\begin{array}{cc}
(1,1) & \left(c_{1}, c_{2}\right) \\
\left(d_{1}, d_{2}\right) & (3,0)
\end{array}\right)
\end{array}
$$

In this game, there are two pure Nash equilibria, namely $(M, L)$ and $(B, R)$, and one fully mixed Nash equilibrium, which gives payoff $\left(\left(3-c_{1}\right)\left(1-c_{1}\right)\left(4-c_{1}-\right.\right.$ $\left.\left.d_{1}\right)^{-1}+c_{1},-c_{2} d_{2}\left(1-c_{2}-d_{2}\right)\right)$. If at stage $m_{0}$, players play $(B, R)$, then the total payoff of Player 2 is smaller than $a_{2} \leq 1 / 2$. If at stage $m_{0}$, players play either $(M, L)$ or the mixed Nash equilibrium, then $m_{0}=1$. Indeed, otherwise Player 1 would have a profitable deviation by playing $M$ at stage 1 .

The above analysis shows that if $\delta \in \Delta_{2}$, there is at most one Nash equilibrium which gives Player 2 a total discounted payoff in $[1 / 2,3 / 4]$ : play the mixed Nash equilibrium at stage 1 .

This is enough to conclude that $E_{\delta}$ and $E_{\delta}^{\prime}$ do not converge as $\delta$ goes to one. In addition, one can easily check that all arguments are robust to small perturbations of the payoffs of the stochastic game.

Remark 2.7. In this example, the set of uniform equilibrium payoffs $E_{\infty}$ corresponds to the three Nash equilibrium payoffs of the one-stage game $(1,1),(3,0)$ and $\left(\left(3-c_{1}\right)\left(1-c_{1}\right)\left(4-c_{1}-d_{1}\right)^{-1}+c_{1},-c_{2} d_{2}\left(1-c_{2}-d_{2}\right)\right)$.

\section{Open questions}

1) Many Folk theorems in the literature require the limit set to have nonempty interior. In both examples, $E_{\delta}=E_{\delta}^{\prime}$ has empty interior for each discount factor. An interesting direction of research would be to investigate whether $\left(E_{\delta}\right)$ and $\left(E_{\delta}^{\prime}\right)$ converge under some interiority conditions on the equilibrium payoff sets.

2) The examples are not robust to the introduction of an extensive-form correlation device ${ }^{8}$. In example 2.1, if the players can publicly observe the

\footnotetext{
${ }^{8}$ An extensive-form correlation device can send a message to each player at each stage of the game.
} 
outcome of a fair coin tossing whenever $k_{3}$ is reached, they can correlate and play $(T, L)$ and $(B, R)$ with probability $1 / 2$. With such correlation device, it is possible to obtain $(1 / 2,1 / 2)$ as an equilibrium payoff for all discount factors. In addition, one can show that example 2.5 is not even robust to the introduction of a normal-form correlation device.

\section{References}

[1] D. Abreu, D. Pearce, and E. Stacchetti. Toward a theory of discounted repeated games with imperfect monitoring. Econometrica, 58(5):1041-1063, 1990.

[2] R. Aumann and L. Shapley. Long-term competition-a game-theoretic analysis. In Essays in Game Theory, pages 1-15. Springer, 1994.

[3] T. Bewley and E. Kohlberg. The asymptotic theory of stochastic games. Mathematics of Operations Research, 1(3):197-208, 1976.

[4] P. Dutta. A folk theorem for stochastic games. Journal of Economic Theory, 66(1):1-32, 1995.

[5] D. Fudenberg and D. Levine. An approximate folk theorem with imperfect private information. Journal of Economic Theory, 54(1):26-47, 1991.

[6] D. Fudenberg, D. Levine, and E. Maskin. The folk theorem with imperfect public information. Econometrica, 62(5):997-1031, 1994.

[7] D. Fudenberg, D. Levine, and S. Takahashi. Perfect public equilibrium when players are patient. Games and Economic Behavior, 61(1):27-49, 2007.

[8] D. Fudenberg and E. Maskin. The folk theorem in repeated games with discounting or with incomplete information. Econometrica, 54(3):533-554, 1986.

[9] D. Fudenberg and Y. Yamamoto. The folk theorem for irreducible stochastic games with imperfect public monitoring. Journal of Economic Theory, 146(4):1664-1683, 2011.

[10] J. Hörner, T. Sugaya, S. Takahashi, and N. Vieille. Recursive methods in discounted stochastic games: An algorithm for $\delta \rightarrow 1$ and a folk theorem. Econometrica, 79(4):1277-1318, 2011.

[11] B. Kocel-Cynk, W. Pawłucki, and A. Valette. A short geometric proof that hausdorff limits are definable in any o-minimal structure. Advances in Geometry, 14(1):49-58, 2014. 
[12] E. Lehrer. Nash equilibria of $n$-player repeated games with semi-standard information. International Journal of Game Theory, 19(2):191-217, 1990.

[13] E. Lehrer. On the equilibrium payoffs set of two-player repeated games with imperfect monitoring. International Journal of Game Theory, 20(3):211-226, 1992.

[14] E. Lehrer. Two-player repeated games with nonobservable actions and observable payoffs. Mathematics of Operations Research, 17(1):200-224, 1992.

[15] J.F. Mertens, S. Sorin, and S. Zamir. Repeated games, volume 55. Cambridge University Press, 2015.

[16] A. Neyman. Real algebraic tools in stochastic games. In Stochastic Games and Applications, pages 57-75. Springer, 2003.

[17] A. Neyman and S. Sorin. Stochastic games and applications. Kluwer Academic Publishers, 2003.

[18] J. Renault and T. Tomala. Communication equilibrium payoffs in repeated games with imperfect monitoring. Games and Economic Behavior, 49(2):313-344, 2004.

[19] J. Renault and T. Tomala. General properties of long-run supergames. Dynamic Games and Applications, 1(2):319-350, 2011.

[20] A. Rubinstein. Equilibrium in supergames. In Essays in Game Theory, pages 17-27. Springer, 1994.

[21] L.S. Shapley. Stochastic games. Proceedings of the National Academy of Sciences of the United States of America, 39(10):1095-1100, 1953.

[22] E. Solan. Three-player absorbing games. Mathematics of Operations Research, 24(3):669-698, 1999.

[23] S Sorin. Asymptotic properties of a non-zero-sum stochastic game. International Journal of Game Theory, 15(2):101-107, 1986.

[24] S. Sorin. On repeated games with complete information. Mathematics of Operations Research, 11(1):147-160, 1986.

[25] N. Vieille. Two-player stochastic games i: A reduction. Israel Journal of Mathematics, 119(1):55-91, 2000.

[26] N. Vieille. Two-player stochastic games ii: The case of recursive games. Israel Journal of Mathematics, 119(1):93-126, 2000.

[27] G. Vigeral. A zero-sum stochastic game with compact action sets and no asymptotic value. Dynamic Games and Applications, 3(2):172-186, 2013. 
[28] Q. Wen. The "folk theorem" for repeated games with complete information. Econometrica, 62(4):949-954, 1994. 